Research Article

\title{
Spatial Information and Performance Evaluation in Coprime Array
}

\author{
Ying Zhou, Dazhuan Xu (D), Chao Shi, and Weilin Tu \\ College of Electronic and Information Engineering, Nanjing University of Aeronautics and Astronautics, Nanjing 211106, China \\ Correspondence should be addressed to Dazhuan Xu; xudazhuan@nuaa.edu.cn
}

Received 6 June 2020; Revised 15 July 2020; Accepted 16 September 2020; Published 28 September 2020

Academic Editor: Junpeng Shi

Copyright (c) 2020 Ying Zhou et al. This is an open access article distributed under the Creative Commons Attribution License, which permits unrestricted use, distribution, and reproduction in any medium, provided the original work is properly cited.

\begin{abstract}
In this paper, the mutual information between the received signals and the source in the coprime linear array is investigated. In Shannon's information theory, the mutual information is used to quantify the reduction in the priori uncertainty of the transmitted message. Similarly, the spatial information in the coprime array is the mutual information between direction of arrival (DOA), source amplitude, and received signals. Such information content is composed of two parts. The first part is DOA information, and the second one is scattering information. In a single source scenario, we derive the theoretical expression and its asymptotic upper bound of DOA information. The corresponding expression of scattering information is also formulated theoretically. Besides, the application of spatial information is discussed. We can obtain the optimal array configuration by maximizing the DOA information of the coprime array. Similarly, the information is also used to quantify the performance difference between the coprime array and uniform array. In addition, the entropy error is employed to evaluate the estimation performance based on spatial information. Numerical simulation of the information content confirms our theoretical analysis. The results in this paper have important guiding significance for the design of the coprime array in the actual environment.
\end{abstract}

\section{Introduction}

In array signal processing, source estimation is a fundamental application and has been widely used in radar, sonar, acoustics, astronomy, wireless communications, medical imaging, and other areas (see, for example, [1-4]). Hence, direction of arrival (DOA) estimation emerges as an active area of research and the main purpose of which is to determine the location of sources [5]. Many high-resolution DOA estimation algorithms have been proposed, especially the subspace-based methods such as the multiple signal classification (MUSIC) algorithm [6] and estimation of signal parameters via the rotational invariance technique (ESPRIT) algorithm [7]. However, these algorithms are invalid when detecting more sources than the number of sensors. To solve this problem, additional sensors are required to increase the achievable number of degrees-offreedom (DOFs), which leads to an increased complexity. Therefore, an active research topic has been focused on how to increase the number of DOFs for DOA estimation.
Nowadays, coprime arrays, a kind of sparse array, have attracted noticeable attention, owing to their superior performance [8]. Compared with a uniform linear array (ULA), a coprime array has a larger aperture with the same number of sensors so that it can acquire a higher accuracy. More importantly, coprime arrays enable to break through the limitation of the DOFs. Motivated by these advantages, a series of efforts have been made to exploit the coprime array for DOA estimation [9]. Furthermore, in [10], the authors introduced the coprime array into the massive MIMO system to alleviate mutual coupling, increase the DOFs, and enhance the spatial resolution. This approach takes full advantage of the coprime array configuration. In [11], a novel sparse reconstruction-based source estimation algorithm was proposed, which considers the estimation accuracy of DOA and power as well as the number of DOFs. The source estimation algorithm enjoys certain performance advantages over other existing algorithms according to multiple evaluation metrics. There are also some studies on two-dimensional (2D) DOA estimation. The authors in [12] 
focused on the coprime property of coprime planar arrays (CPPAs), and the sparse array extension model with the sum-difference coarray was derived. Further, they proposed the aperture extension based 2D DOA estimation method with CPPAs via the improved sparse representation algorithm. The cases extending the sparse array extension model for MIMO radars were also discussed in [12].

Based on the derived difference coarray, a super-resolution estimation algorithm was proposed in [13] by applying the spatial smoothing technique. The algorithm is able to identify more sources than sensors. However, there may exist several spurious peaks in the estimated spatial spectrum, which will dramatically affect the overall estimation performance. In this sense, it still remains a challenging problem to perform accurate DOA estimation in the coprime array. Recently, some novel high-resolution coprime array DOA estimation algorithms are proposed. In [14], the algorithm is based on the virtual array interpolation and makes full use of the information received by the coprime array, so it has advantages in estimation performance. In [15], a coprime array interpolation approach to provide an off-grid DOA estimation was proposed.

The information theory [16] is the theoretical basis of communication technology, and the sensor arrays' system has a profound internal relationship with the communication system. By observing the received signal of the array, we can obtain information about the source, such as DOA, source amplitude, and so on. From the information theory point of view, mutual information is used to quantify the information about unknown parameters provided by the observation of the output. In light of certain common aspects between the information theory and source estimation problems, it is fairly reasonable to look at these two different areas from a unified perspective. At present, the research based on Shannon information theory for array signal processing mainly focuses on the detection of the number of signals [17]. Many approaches were proposed such as Akaike information criterion (AIC) [18], minimum description length (MDL) criterion [19], and effective detection criterion [20-22]. To the best of our knowledge, only a few researchers employ the information theory to address the performance analysis of the DOA estimation, without studying the amount of information obtained from the multisensor array.

In [23], the sensor array information acquisition process was studied for the first time and the initial definition of spatial information is presented therein. However, the author's research was aimed at the ULA, and there is no application of information theory methods on other array models. So, we apply the framework of spatial information to the coprime array.

In this paper, information theory is used to characterize the estimation process in the coprime array system. Here, we just consider the single source case in the system model. Although the derivation of theoretical expressions in this paper is for the case of a single source, it is also applicable to the sparse multiple-source case where the sources do not interfere with each other and each of them can be analysed individually as a single source. It is difficult to analyse a multiple-source case where they are close to each other, even in a ULA system. For adjacent multiple sources, the posterior probability density is multidimensional, so the computation is also very large in numerical simulation. Therefore, in this paper, we only consider the simplest case at present. In the following research, this system model will be gradually extended to a more general multiple-source case. The main contributions of this study are demonstrated as follows.

Firstly, the corresponding theoretical expressions of DOA information and scattering information are derived in the existence of complex additive white Gaussian noise when the source is single. The regularity of information change reflects the information acquisition efficiency of a coprime array system and may provide a guidance for system designers. Secondly, the asymptotic upper bound of DOA information is also presented. It is concluded that this upper bound is consistent with CRB at high SNR, determining the maximum accuracy of the estimation. Thirdly, the application of DOA information is discussed. The optimal array configuration can be obtained by maximizing the DOA information of the coprime array. Similarly, we use the asymptotic upper bound of DOA information for the comparison between the coprime array and uniform array. The performance difference between the two models is thus quantified by the difference of the amount of information. For the sake of evaluating information acquisition capability of the coprime array system from the perspective of information theory, we propose an evaluation index entropy error in light of the observation interval and the amount of information. We note that it reflects the dispersion of data set and the accuracy of estimation. It also proves that entropy error tends to CRB in the high SNR region.

This paper is organized as follows. The system model of a coprime linear array is presented, and some basic assumptions on priori probability distributions are introduced in Section 2. In Section 3, the expression of DOA information and the asymptotic upper bound are derived. The applications of DOA information are discussed in Section 4. The scattering information is studied, and the corresponding theoretical expression is given in Section 5. The proposed concept is tested via a few simulations, which appear in Section 6. The main results of this paper are discussed and concluded in Section 7.

\section{System Model}

Let us consider a general coprime linear array (CLA) made up of two uniform linear arrays, as shown in Figure 1. Subarray 1 has $M_{1}$ sensors spaced $M_{2} d$ apart and subarray 2 has $M_{2}$ sensors spaced $M_{1} d$ apart. Here, $M_{1}$ and $M_{2}$ are the coprime integers (generally assuming $M_{1}<M_{2}$ ) and $d$ is a half wavelength, i.e., $d=(\lambda / 2)$. Assuming a single far-field narrow-band source is impinging from direction $\theta$, the received signals can be modeled as

$$
\mathbf{x}(t)=\mathbf{a}(\theta) s(t)+\mathbf{w}(t),
$$

where $s(t)$ denotes the signal waveform and $\mathbf{w}(t) \sim$ $C N\left(0, N_{0} \mathbf{I}\right)$ represents the independent and identically 


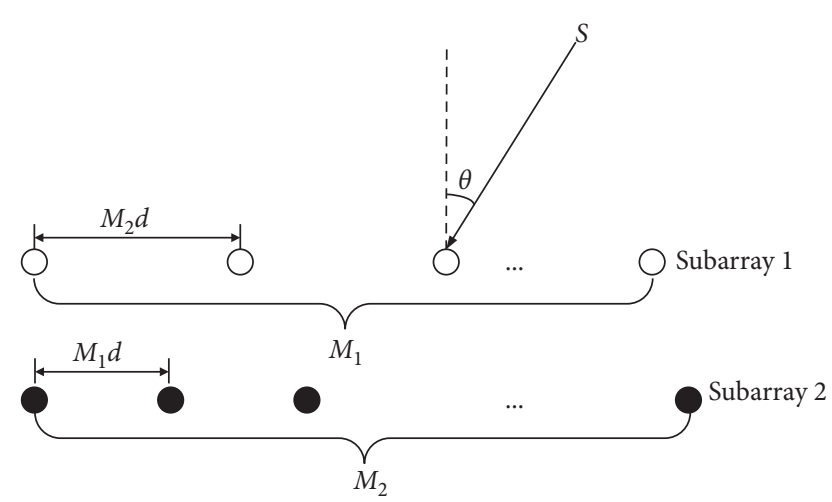

Figure 1: Topological structure of the coprime linear array.

distributed zero-mean additive white Gaussian noise vector. Here, $N_{0}$ denotes the noise power. $\mathbf{a}(\theta)$ represents the steering vector and the specific expression is

$$
\mathbf{a}(\theta)=\left[1, e^{-j(2 \pi / \lambda) q_{1} d \sin \theta}, \ldots, e^{-j(2 \pi / \lambda)(M-1) q_{M-1} d \sin \theta}\right]^{T},
$$

where $q_{i} d$ denotes the position of the $i$-th sensor and the total number of elements is $M . \lambda$ is the carrier wavelength. The directional vector of subarray 1 and subarray 2 , respectively, are

$$
\begin{aligned}
& \mathbf{a}_{1}(\theta)=\left[1, e^{-j(2 \pi / \lambda) M_{2} d \sin \theta}, \ldots, e^{-j(2 \pi / \lambda)\left(M_{1}-1\right) M_{2} d \sin \theta}\right]^{T}, \\
& \mathbf{a}_{2}(\theta)=\left[1, e^{-j(2 \pi / \lambda) M_{1} d \sin \theta}, \ldots, e^{-j(2 \pi / \lambda)\left(M_{2}-1\right) M_{1} d \sin \theta}\right]^{T} .
\end{aligned}
$$

According to equations (3) and (4), the total directional vector is

$$
\mathbf{a}(\theta)=\left[\mathbf{a}_{1}^{T}(\theta), \mathbf{a}_{21}^{T}(\theta)\right]^{T},
$$

where $\mathbf{a}_{21}^{T}(\theta)$ is the new direction vector formed by removing the first row of $\mathbf{a}_{2}^{T}(\theta)$.

Considering a single snapshot scenario, omitting time $t$, we can rewrite (1) as

$$
\mathbf{x}=\mathbf{a}(\theta) s+\mathbf{w}
$$

where $s=\alpha e^{j \varphi}, \alpha$ is the constant, and $\varphi$ is uniformly distributed. The received signal is mainly related to the DOA $\theta$ and the source $s . \theta$ is continuous uniformly distributed in the interval.

Next, we introduce a priori probability density function about the direction of angle $\theta$ and the phase $\varphi$ that will be used in the following.

$\theta$ is uniformly distributed in the interval $[-(\|\Theta\| / 2),(\|\Theta\| / 2)]$, where $\|\Theta\|$ is the observation range. The priori probability of $\theta$ is, therefore, given by

$$
p(\theta)=\frac{1}{\|\Theta\|},
$$

where $\varphi$ is uniformly distributed in the interval $[0,2 \pi]$, so the priori probability of $\varphi$ is given by

$$
p(\varphi)=\frac{1}{2 \pi} \text {. }
$$

Here, we define the source signal-to-noise ratio (SNR) as

$$
\rho^{2}=\frac{\alpha^{2}}{N_{0}}
$$

where $\alpha^{2}$ is the power of the useful signal and $N_{0}$ represents the power of the noise. $\rho^{2}$ is an important parameter which constantly recurs in the remainder of this paper.

According to the above assumption, we will be concerned with the spatial information in the following sections. In [23], the definition of spatial information is given for the first time. The spatial information is expressed as the sum of the DOA information $I(\mathbf{x} ; \theta)$ and the scattering information $I(\mathbf{x} ; s \mid \theta)$.

\section{DOA Information}

In this section, we focus on the DOA information, and the actual value of DOA is $\theta_{0}$. We provide the general expression and its asymptotic upper bound. CRB of DOA estimation is also studied further.

3.1. General Expression. In order to obtain the DOA information, the central problem is to form the probability distribution of DOA. Our analytical approach is to fix on the typical received signals resulted from the actual value of DOA and to consider the distribution of the estimated value which could have produced it.

Considering $\mathbf{w}$ is a complex Gaussian vector, the multidimensional probability density of $\mathbf{x}$ conditioned on $\theta$ and $\varphi$ is given by

$$
\begin{aligned}
p(\mathbf{x} \mid \theta, \varphi)= & \left(\frac{1}{\pi N_{0}}\right)^{M} \exp \left(-\frac{1}{N_{0}}(\mathbf{x}-\mathbf{a}(\theta) s)^{H}(\mathbf{x}-\mathbf{a}(\theta) s)\right) \\
= & \left(\frac{1}{\pi N_{0}}\right)^{M} \exp \left(-\frac{1}{N_{0}}\left(\mathbf{x}^{H} \mathbf{x}+M \alpha^{2}\right)\right) \\
& \cdot \exp \left(\frac{2 \alpha}{N_{0}} \operatorname{Re}\left(e^{-j \varphi} \mathbf{a}^{H}(\theta) \mathbf{x}\right)\right)
\end{aligned}
$$

where $\operatorname{Re}(\cdot)$ denotes taking the real part of a complex number. Then the joint probability density of $\mathbf{x}$ and $\theta$ conditioned on $\varphi$ is derived as

$$
p(\mathbf{x}, \theta \mid \varphi)=p(\theta) p(\mathbf{x} \mid \theta, \varphi) .
$$

According to the probability theory, we have the joint probability distribution of $\mathbf{x}$ and $\theta$ as

$$
p(\mathbf{x}, \theta)=\int_{0}^{2 \pi} p(\mathbf{x}, \theta \mid \varphi) p(\varphi) \mathrm{d} \varphi .
$$


Then, we have the posteriori probability density function in (13). The term $\mathbf{x}^{H} \mathbf{x}$ disappears because it depends on the true values instead of the unknown parameters. Note that the denominator is a normalizing constant uncorrelated with the parameters; thus, the shape of the probability distribution is mainly determined by the numerator:

$$
p(\theta \mid \mathbf{x})=\frac{p(\mathbf{x}, \theta)}{\int_{-(\|\Theta / / 2\|)}^{\|\Theta\| / 2} p(\mathbf{x}, \theta) \mathrm{d} \theta}=\frac{\int_{0}^{2 \pi} \exp \left(\left(2 \alpha / N_{0}\right) \operatorname{Re}\left(e^{-j \varphi} \mathbf{a}^{H}(\theta) \mathbf{x}\right)\right) \mathrm{d} \varphi}{\int_{-(\|\Theta\| / 2)}^{\|\Theta\| / 2} \int_{0}^{2 \pi} \exp \left(\left(2 \alpha / N_{0}\right) \operatorname{Re}\left(e^{-j \varphi} \mathbf{a}^{H}(\theta) \mathbf{x}\right)\right) \mathrm{d} \varphi \mathrm{d} \theta} .
$$

We further have

$$
\begin{aligned}
& \int_{0}^{2 \pi} \exp \left(\frac{2 \alpha}{N_{0}} \operatorname{Re}\left(e^{-j \varphi} \mathbf{a}^{H}(\theta) \mathbf{x}\right)\right) \mathrm{d} \varphi \\
& \quad=\int_{0}^{2 \pi} \exp \left(\frac{2 \alpha}{N_{0}}\left(\operatorname{Re}\left(\mathbf{a}^{H}(\theta) \mathbf{x}\right) \cos \varphi+\operatorname{Im}\left(\mathbf{a}^{H}(\theta) \mathbf{x}\right) \sin \varphi\right)\right) \mathrm{d} \varphi \\
& \quad=2 \pi I_{0}\left(\frac{2 \alpha}{N_{0}}\left|\mathbf{a}^{H}(\theta) \mathbf{x}\right|\right),
\end{aligned}
$$

where $\operatorname{Im}(\cdot)$ denotes the imaginary part of a complex number and $I_{0}(\cdot)$ denotes the zero-order modified Bessel function of the first kind. Substituting (14) into (13), we can get the following expression:

$$
p(\theta \mid \mathbf{x})=\frac{I_{0}\left(\left(2 \alpha / N_{0}\right)\left|\mathbf{a}^{H}(\theta) \mathbf{x}\right|\right)}{\int_{-(\|\Theta\| / 2)}^{\|\Theta\| / 2} I_{0}\left(\left(2 \alpha / N_{0}\right)\left|\mathbf{a}^{H}(\theta) \mathbf{x}\right|\right) \mathrm{d} \theta} .
$$

We are concerned about how much information we can obtain from the posteriori probability density function. Since the posteriori probability density of $\theta$ is given, the quantity of DOA information is the difference of the entropies of the priori and posteriori probability distributions based on the mutual information formula, i.e.,

$$
\begin{aligned}
I(\mathbf{x} ; \theta) & =h(\theta)-h(\theta \mid \mathbf{x}) \\
& =\log _{2}\|\Theta\|+E_{\mathbf{x}}\left[\int_{-(\|\Theta\| / 2)}^{\|\Theta\| / 2} p(\theta \mid \mathbf{x}) \log _{2} p(\theta \mid \mathbf{x}) \mathrm{d} \theta\right] .
\end{aligned}
$$

Although equation (16) is difficult to solve, we can figure out the results through numerical simulation. The asymptotic expression under the specific condition of high SNR is also presented in the following section.

3.2. Asymptotic Upper Bound. Considering the actual direction of received signals is $\theta_{0}$ and $s=\alpha e^{j \varphi_{0}}$, we can rewrite (6) as

$$
\mathbf{x}=\alpha e^{j \varphi_{0}} \mathbf{a}\left(\theta_{0}\right)+\mathbf{w}
$$

Substituting it into (15) yields another form of the posteriori probability density function conditioned on noise

$$
p(\theta \mid \mathbf{w})=\frac{I_{0}\left(\left(2 \alpha / N_{0}\right)\left|\alpha \mathbf{a}^{H}(\theta) \mathbf{a}\left(\theta_{0}\right)+F_{\mathbf{w}}\right|\right)}{\int_{-(\|\Theta\| / 2)}^{\|\Theta\| / 2} I_{0}\left(\left(2 \alpha / N_{0}\right)\left|\alpha \mathbf{a}^{H}(\theta) \mathbf{a}\left(\theta_{0}\right)+F_{\mathbf{w}}\right|\right) \mathrm{d} \theta} .
$$

Note that $F_{\mathbf{w}}=e^{-j \varphi_{0}} \mathbf{a}^{H}(\theta) \mathbf{w}$ is the noise term. Since $\mathbf{w}$ is a complex random quantity, the phase $e^{-j \varphi_{0}}$ may be absorbed into it without altering its statistical properties and is omitted in the following analysis. It can be seen from equation (18) that the characteristics of $p(\theta \mid \mathbf{w})$ depend markedly on the contributions of the signal and noise to the probability distribution.

In the case of high SNR, the signal plays a dominant role. We can neglect the noise term without changing the characteristics of the posteriori distribution. Thus, we obtain

$$
p(\theta \mid \mathbf{w})=\frac{I_{0}\left(2 \rho^{2}\left|\mathbf{a}^{H}(\theta) \mathbf{a}\left(\theta_{0}\right)\right|\right)}{\int_{-(\|\Theta\| / 2)}^{\|\Theta\| / 2} I_{0}\left(2 \rho^{2}\left|\mathbf{a}^{H}(\theta) \mathbf{a}\left(\theta_{0}\right)\right|\right) \mathrm{d} \theta} .
$$

According to the specific expression of the steering vector, we have the expression of $\left|\mathbf{a}^{H}(\theta) \mathbf{a}\left(\theta_{0}\right)\right|$ in (20), where $\omega=\left(\pi d\left(\sin \theta-\sin \theta_{0}\right) / \lambda\right)$.

$$
\begin{aligned}
& \begin{aligned}
\left|\mathbf{a}^{H}(\theta) \mathbf{a}\left(\theta_{0}\right)\right| & =\left|\exp \left(-j \omega M_{2}\left(M_{1}-1\right)\right) \frac{\sin \left(\omega M_{1} M_{2}\right)}{\sin \left(\omega M_{2}\right)}+\exp \left(-j \omega M_{1}\left(M_{2}-1\right)\right) \frac{\sin \left(\omega M_{1} M_{2}\right)}{\sin \left(\omega M_{1}\right)}-1\right| \\
& =\sqrt{\frac{\sin ^{2}\left(\omega M_{1} M_{2}\right)}{\sin ^{2}\left(\omega M_{2}\right)}+\frac{\sin ^{2}\left(\omega M_{1} M_{2}\right)}{\sin ^{2}\left(\omega M_{1}\right)}+2 \frac{\sin ^{2}\left(\omega M_{1} M_{2}\right)}{\sin \left(\omega M_{1}\right) \sin \left(\omega M_{2}\right)} \cos \left(\omega\left(M_{1}-M_{2}\right)\right)+1-2\left(\cos \left(\omega M_{2}\left(M_{1}-1\right) \frac{\sin \left(\omega M_{1} M_{2}\right)}{\sin \left(\omega M_{2}\right)}+\cos \left(\omega M_{1}\left(M_{2}-1\right)\right) \frac{\sin _{(}\left(\omega M_{1} M_{2}\right)}{\sin \left(\omega M_{1}\right)}\right)\right.}
\end{aligned} \\
& \text { In order to extract the approximation of DOA infor- } \quad\left|\mathbf{a}^{H}(\theta) \mathbf{a}\left(\theta_{0}\right)\right| \approx M-\gamma^{2}\left(\theta-\theta_{0}\right)^{2},
\end{aligned}
$$
mation, we exploit the Taylor series expansion at $\sin \theta=$ $\sin \theta_{0}$ on $\left|\mathbf{a}^{H}(\theta) \mathbf{a}\left(\theta_{0}\right)\right|$ as where the specific expression of $\gamma^{2}$ is given by (22) and the higher order term of $\left(\theta-\theta_{0}\right)$ is neglected, due to the fact that 
the DOA is in the vicinity of the true direction $\theta_{0}$ when SNR is high:

$$
\gamma^{2}=\frac{\left(M_{1}^{4} M_{2}^{2}-4 M_{1}^{3} M_{2}^{2}+2 M_{1}^{3} M_{2}^{3}+2 M_{1}^{3} M_{2}+M_{1}^{2} M_{2}^{4}-4 M_{1}^{2} M_{2}^{3}+4 M_{1}^{2} M_{2}^{2}-2 M_{1}^{2} M_{2}+2 M_{1} M_{2}^{3}-2 M_{1} M_{2}^{2}\right)}{6 M} \frac{\pi^{2} d^{2} \cos ^{2} \theta_{0}}{\lambda^{2}} .
$$

Moreover, the asymptotic expansion for $I_{0}$ is

$$
I_{0}(x) \sim \frac{e^{x}}{\sqrt{2 \pi x}}\left\{1+\frac{1}{8 x}+o\left(\frac{1}{x^{2}}\right)\right\} .
$$

Substituting (21) and (23) into (19), we can derive

$$
\begin{aligned}
p(\theta \mid \mathbf{w}) & \approx \kappa \exp \left(-2 \rho^{2} \gamma^{2}\left(\theta-\theta_{0}\right)^{2}\right) \\
& \approx \frac{1}{\sqrt{2 \pi \sigma^{2}}} \exp \left(-\frac{\left(\theta-\theta_{0}\right)^{2}}{2 \sigma^{2}}\right),
\end{aligned}
$$

where $\kappa$ denotes the normalized constant coefficient and the posteriori distribution is approximately Gaussian near $\theta_{0}$. The corresponding variance is given by

$$
\sigma^{2}=\left(4 \rho^{2} \gamma^{2}\right)^{-1}
$$

Based on the derivation of the differential entropy in a Gaussian scenario [24], the asymptotic upper bound of DOA information can be formulated as

$$
\begin{aligned}
I(\mathbf{x} ; \theta) & =h(\theta)-h(\theta \mid \mathbf{x})=\log _{2}\|\Theta\|-\frac{1}{2} \log _{2}\left(2 \pi e \sigma^{2}\right) \\
& =\log _{2}\left(\frac{\|\Theta\|}{\sqrt{2 \pi e \sigma^{2}}}\right) .
\end{aligned}
$$

3.3. Cramér-Rao Bound. In estimation theory, the Cramér-Rao bound (CRB) is a significant evaluating indicator for the performance of unbiased estimators. It provides a lower bound for the mean square error (MSE) of the estimators. In [25], the authors derive the CRB for the unbiased estimator of $\theta$ as

$$
\sigma_{\mathrm{CRB}}^{2}=\frac{N_{0}}{2}\left\{\operatorname{Re}\left[s^{H} \mathbf{D}^{H}(\theta) \prod_{a}^{\perp} \mathbf{D}(\theta) s\right]\right\}^{-1}
$$

where

$$
\begin{aligned}
\mathbf{D}(\theta) & =\frac{\partial \mathbf{a}(\theta)}{\partial \theta}, \\
\prod_{\mathbf{a}}^{\perp} & =I-\mathbf{a}(\theta)\left(\mathbf{a}^{H}(\theta) \mathbf{a}(\theta)\right)^{-1} \mathbf{a}^{H}(\theta) .
\end{aligned}
$$

Clearly, in the case of the model in this paper, we have $\mathbf{a}^{H}(\theta) \mathbf{a}(\theta)=M$

$$
\begin{aligned}
\mathbf{D}^{H}(\theta) \mathbf{D}(\theta)= & \frac{4 \pi^{2} d^{2} \cos ^{2} \theta}{\lambda^{2}}\left(M_{2}^{2} \frac{M_{1}\left(M_{1}-1\right)\left(2 M_{1}-1\right)}{6}\right. \\
& \left.+M_{1}^{2} \frac{M_{2}\left(M_{2}-1\right)\left(2 M_{2}-1\right)}{6}\right), \\
\mathbf{D}^{H}(\theta) \mathbf{a}(\theta)= & -j \frac{\pi d \cos \theta}{\lambda} M_{1} M_{2}\left(M_{1}+M_{2}-2\right) .
\end{aligned}
$$

Substituting (29) into (27), we obtain the CRB for DOA estimation in the coprime array as shown in (30). Then according to the specific expression of $\gamma^{2}$ in (22), we can simplify the expression of equation (30) as shown in (31). The expression of CRB in (31) is completely the same as (25). It indicates that, as a lower bound of MSE, CRB implies the upper bound of DOA information as well in the high SNR region. Therefore, the posteriori entropy $h(\theta \mid \mathbf{x})$ can be used for evaluating the performance of the estimation.

$$
\begin{aligned}
& \sigma_{\mathrm{CRB}}^{2}=\left(\frac{2}{3} \rho^{2} \frac{\left.\left(M_{1}^{4} M_{2}^{2}-4 M_{1}^{3} M_{2}^{2}+2 M_{1}^{3} M_{2}^{3}+2 M_{1}^{3} M_{2}+M_{1}^{2} M_{2}^{4}-4 M_{1}^{2} M_{2}^{3}+4 M_{1}^{2} M_{2}^{2}-2 M_{1}^{2} M_{2}+2 M_{1} M_{2}^{3}-2 M_{1} M_{2}^{2}\right) \frac{\pi^{2} d^{2} \cos ^{2} \theta}{\lambda^{2}}\right)^{-1}}{\sigma_{\mathrm{CRB}}^{2}}=\left(4 \rho^{2} \gamma^{2}\right)^{-1} .\right.
\end{aligned}
$$

\section{The Application of DOA Information}

4.1. Optimal Array Configuration. Since the mutual information between the received signals and DOA represents the uncertainty reduction of the DOA estimation conditioned on the known received signals, the more DOA information obtained means the higher accuracy of DOA estimation. Thus, we can optimize the array configuration for CLA to maximize the DOA information obtained.

Here, we consider the same total number of elements $M$. In the expression of the asymptotic upper bound of DOA information, the positions of $M_{1}$ and $M_{2}$ are 
interchangeable. Therefore, equation (26) takes the maximum value when the number of elements of two subarrays is the same. It is clear that on the premise that $M_{1}$ and $M_{2}$ are the coprime integers, the closer the two numbers are, the better the performance of the array will be.

4.2. Comparison between Coprime Array and Uniform Array. Similarly, we use the asymptotic upper bound of DOA information for comparison between the coprime array and uniform array.

In [23], the asymptotic upper bound of DOA information in the ULA in the high SNR region is

$$
I_{u}(\mathbf{x} ; \theta)=\log _{2}\|\Theta\|-\frac{1}{2} \log _{2}\left(2 \pi e \sigma_{u}^{2}\right),
$$

where

$$
\sigma_{u}^{2}=\left(\frac{2}{3} \rho^{2} M\left(M^{2}-1\right) \frac{\pi^{2} d^{2} \cos ^{2} \theta_{0}}{\lambda^{2}}\right)^{-1} .
$$

Here, we consider the most extreme case for the array configuration of CLA; that is, $M_{1} \approx M_{2} \approx((M+1) / 2)$. In this case, the difference between the asymptotic upper bound of DOA information of the two array models is

$$
\begin{aligned}
I_{c}(\mathbf{x} ; \theta)-I_{u}(\mathbf{x} ; \theta) \\
\quad=\frac{1}{2} \log _{2}\left(\frac{6 \gamma^{2} \lambda^{2}}{M\left(M^{2}-1\right) \pi^{2} d^{2} \cos ^{2} \theta}\right) \\
\quad \approx \frac{1}{2} \log _{2}\left(\frac{4 M_{1}^{6}-8 M_{1}^{5}+8 M_{1}^{4}-4 M_{1}^{3}}{M^{2}\left(M^{2}-1\right)}\right) \\
\quad \approx \frac{1}{2} \log _{2}\left(\frac{M^{2}}{16}-\frac{M}{4}+\frac{1}{2}\right) .
\end{aligned}
$$

The above equation is the result of quantifying the performance difference between the two array models.

4.3. Entropy Error. The previous analysis provides some guidelines for the application of DOA information to the estimation problems. It follows that the posteriori entropy $h(\theta \mid \mathbf{x})$ represents the uncertainty of the unknown parameters and can be used for evaluating the performance of the estimation. As SNR increases, the posteriori entropy continues to decline, indicating that the estimation performance is getting better.

Therefore, the definition of entropy error (EE) is put forth as an evaluation index to accurately assess the estimation performance in [23]. Although the array model in [23] is a ULA, this evaluation index is also applicable to the CLA in this paper. The specific expression of EE is

$$
\sigma_{\mathrm{EE}}^{2}=\frac{2^{2 h(\theta \mid \mathbf{x})}}{2 \pi e}=\frac{1}{2 \pi e} \frac{\|\Theta\|^{2}}{2^{2 I(\mathbf{x} ; \theta)}},
$$

where $I(\mathbf{x} ; \theta)$ is obtained in (16).
Note that EE is independent of the specific parameter estimation method. Then, it will provide a basis for comparing the performance of different estimation algorithms.

Furthermore, from (26) and (35), we can obtain the lower bound of EE in the case of high SNR

$$
\sigma_{\mathrm{EE}}^{2} \geq\left(4 \rho^{2} \gamma^{2}\right)^{-1} \text {. }
$$

This equation reflects that $\mathrm{EE}$ tends to $\mathrm{CRB}$ in the high SNR region.

We can learn better about the proposed entropy error from information theory. By Shannon's theorem for the noisy channel, we are allowed to transmit $N=2^{I(\theta ; \mathbf{x})}$ distinguishable symbols without any error. That is, assuming that the observation interval has been partitioned into $N$ equiprobable subsets, we are able to assign the parameter $\theta$ to its proper subset based on observing $\mathbf{x}$, generated by the measurement process.

In (35), the entropy error is only related to DOA information and the observation range. When the DOA information increases by 1 bit, the entropy error becomes a quarter of the original value. Similarly, when the observation range is reduced by half, it is the same thing as multiplying the entropy error by a quarter. Therefore, the effect of the increase of 1 bit in DOA information and the reduction of the observation range by half is the same.

In conclusion, the greater the mutual information, the smaller the entropy error and the more accurately we can estimate the parameter characterizing the entity we are trying to measure.

\section{Scattering Information}

In this section, the scattering information is analysed under the condition that the amplitude $\alpha$ is constant. In this case, the scattering information is equivalent to the phase information.

Similar to the analysis of the DOA information, the central problem is to form a posteriori probability density function of the phase conditioned on the observation vector $\mathbf{x}$ and the parameter $\theta$. Based on the Bayes formula, the posteriori probability density function is presented as

$$
p(\varphi \mid \mathbf{x}, \theta)=\frac{p(\mathbf{x}, \varphi \mid \theta)}{p(\mathbf{x} \mid \theta)}=\frac{p(\mathbf{x} \mid \theta, \varphi)}{\int_{0}^{2 \pi} p(\mathbf{x} \mid \theta, \varphi) \mathrm{d} \varphi} .
$$

Substituting (10) into (37) and ignoring the constant term, we have

$$
p(\varphi \mid \mathbf{x}, \theta)=\frac{\exp \left(\left(2 \alpha / N_{0}\right) \operatorname{Re}\left(e^{-j \varphi} \mathbf{a}^{H}(\theta) \mathbf{x}\right)\right)}{\int_{0}^{2 \pi} \exp \left(\left(2 \alpha / N_{0}\right) \operatorname{Re}\left(e^{-j \varphi} \mathbf{a}^{H}(\theta) \mathbf{x}\right)\right) \mathrm{d} \varphi} .
$$

Using the definition of the Bessel function $I_{0}(\cdot)$ as specified in (14), we can obtain that

$$
p(\varphi \mid \mathbf{x}, \theta)=\frac{\exp \left(\left(2 \alpha / N_{0}\right) \operatorname{Re}\left(e^{-j \varphi} \mathbf{a}^{H}(\theta) \mathbf{x}\right)\right)}{2 \pi I_{0}\left(\left(2 \alpha / N_{0}\right)\left|\mathbf{a}^{H}(\theta) \mathbf{x}\right|\right)} .
$$


In addition, substituting the actual observation vector $\mathbf{x}$ as in (17) into (39), we have

$$
p(\varphi \mid \mathbf{w}, \theta)=\frac{\exp \left(2 \rho^{2} \operatorname{Re}\left(e^{-j \varphi} \mathbf{a}^{H}(\theta) \mathbf{a}\left(\theta_{0}\right)+e^{-j \varphi} F_{\mathbf{w}}\right)\right)}{2 \pi I_{0}\left(2 \rho^{2}\left|\mathbf{a}^{H}(\theta) \mathbf{a}\left(\theta_{0}\right)+F_{\mathbf{w}}\right|\right)} .
$$

Then, the scattering information is given by

$$
\begin{aligned}
I(\mathbf{x} ; \varphi \mid \theta) & =h(\varphi \mid \theta)-h(\varphi \mid \mathbf{x}, \theta) \\
& =\log _{2}(2 \pi)+E_{\mathbf{x}, \theta}\left[\int_{0}^{2 \pi} p(\varphi \mid \mathbf{x}, \theta) \log _{2} p(\varphi \mid \mathbf{x}, \theta) \mathrm{d} \varphi\right] .
\end{aligned}
$$

From the equation, we can see that the scattering information depends on the value of the DOA when the amplitude $\alpha$ is constant. This is a general conclusion. It indicates that we have to determine the approximate direction before we estimate the scattering properties.

\section{Numerical Results}

In this section, we provide some simulation results to confirm our theoretical analysis in this paper. In the following simulation, we assume the single source locates in the far field with the true direction $\theta_{0}=0^{\circ}$. In addition, the constant amplitude $\alpha=1$ is used.

Figure 2 depicts the comparison of DOA information between a ULA and a CLA. Here, the number of elements of the uniform array $M$ is set as 10 . In the coprime array, $M_{1}=$ 5 and $M_{2}=6$. The parameter setting ensures that the total number of elements in both arrays is the same. In this figure, the curve of DOA information of the coprime array is drawn according to equation (16) and that of uniform array is based on equation (46) in [23]. All of these results are computed in 10000 independent simulation runs. Clearly, the information is approximately zero in the case of low SNR. This is due to the fact that the conditional entropy will not exceed a priori entropy, and the amount of information is nonnegative. Thus, its lower bound is definitely zero. It also shows that the power of Gaussian noise is much more significant than that of the useful signal in the low SNR region. It is difficult to locate the source from the noise, and we can obtain little information through the observation. With the increase of SNR, the amount of information increases; thus, the DOA is easy to be estimated accurately. When the SNR is $5 \mathrm{~dB}$, the result of the theoretical expression of DOA information coincides with the upper bound obtained by equation (26). This phenomenon indicates the correctness of our derivation. Furthermore, we can find that the DOA information obtained by the coprime array is 1.469 bit more than that obtained by the uniform array when the SNR is high, which is consistent with the theoretical result of 1.4808 bit obtained by equation (34).

Moreover, in order to point out the directive significance of the proposed evaluation index, we compare the theoretical result with the spatially smoothed MUSIC algorithm (SS MUSIC) in [13]. Consistent with the previous simulation parameter, the total number of physical elements is set as 10 .

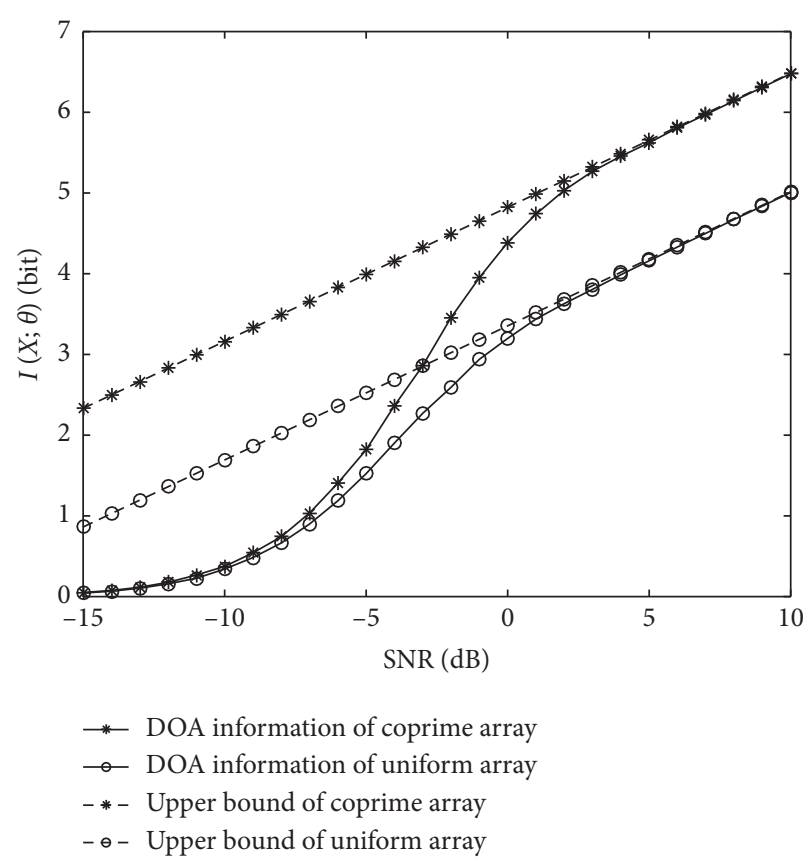

FIGURE 2: DOA information and the asymptotic upper bound for different arrays.

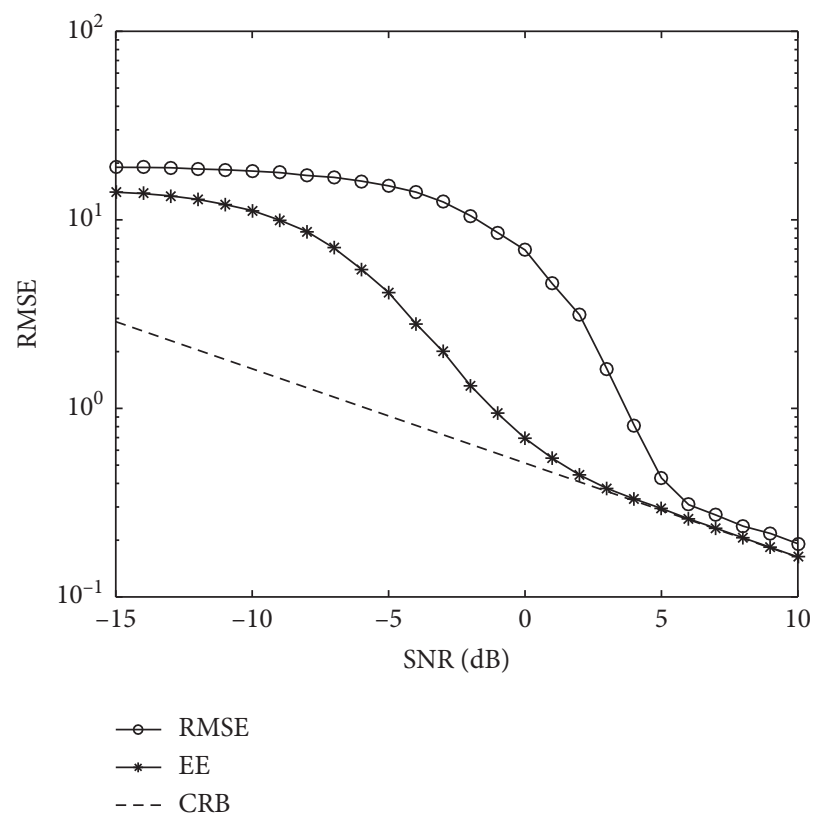

FIgure 3: Comparison among RMSE, EE, and CRB.

Figure 3 shows the comparison among the root mean square error (RMSE) of the actual DOA estimation algorithm, the square root of EE proposed in equation (35), and the square root of CRB. It is illustrated from the figure that EE performs better than RMSE obtained through the algorithm. EE can be computed so long as the posteriori probability distribution is given, thus providing an algorithm independent bound. Besides, in the high SNR region, EE approaches the CRB, verifying the effectiveness of our theoretical analysis in this 


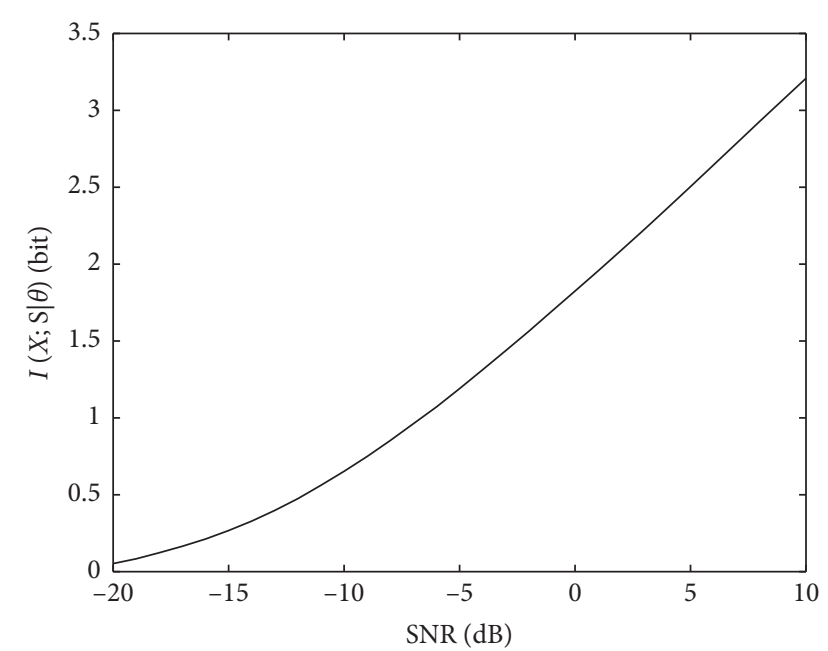

FIGURE 4: Scattering information versus SNR.

paper. However, the RMSE of the SS MUSIC algorithm does not achieve the CRB when the SNR is high. This phenomenon is consistent with the simulation results in [26].

Figure 4 shows the scattering information versus SNR when $M_{1}=5$ and $M_{2}=6$. It is noted that the information grows with SNR increasing, which means we can learn better about the source of interest.

\section{Conclusion}

In this paper, the spatial information in the CLA is investigated. In a single-source scenario, we derive the theoretical expression of both the DOA information and the scattering information. Furthermore, the asymptotic upper bound of the DOA information is derived, and the numerical results confirm its effectiveness. Moreover, the application of DOA information is also discussed. We obtain the optimal array configuration by maximizing the DOA information of the coprime array. Similarly, we use the asymptotic upper bound of DOA information for the comparison between the coprime array and uniform array. In addition, EE is employed as another performance metric to evaluate the information acquisition capability of the coprime array system. When SNR is high, it approaches to CRB. Finally, we can generalize our research to a more complicated scenario, such as extended source amplitude models and multiplesource estimation especially the case when the number of sources is larger than the number of sensors in the array. All these problems are worthy of further investigations.

\section{Data Availability}

The simulation data used to support the findings of this study are included within the article.

\section{Conflicts of Interest}

The authors declare that they have no conflicts of interest.

\section{Acknowledgments}

This work was supported by the National Natural Science Foundation of China (grant number 61971217) and Foundation of the Graduate Innovation Center, Nanjing University of Aeronautics and Astronautics (China) (grant number kfjj20190411).

\section{References}

[1] R. Levanda and A. Leshem, "Synthetic aperture radio telescopes," IEEE Signal Processing Magazine, vol. 27, no. 1, pp. 14-29, 2010.

[2] Y. Wang, X. Ma, C. Chen, and X. Guan, "Designing dual-tone radio interferometric positioning systems," IEEE Transactions on Signal Processing, vol. 63, no. 6, pp. 1351-1365, 2015.

[3] G. Wang, J. Xin, J. Wang, N. Zheng, and A. Sano, "Subspacebased two-dimensional direction estimation and tracking of multiple targets," IEEE Transactions on Aerospace and Electronic Systems, vol. 51, no. 2, pp. 1386-1402, 2015.

[4] X. Zhang, L. Xu, L. Xu, and D. Xu, "Direction of departure (DOD) and direction of arrival (DOA) estimation in MIMO radar with reduced-dimension music," IEEE Communications Letters, vol. 14, no. 12, pp. 1161-1163, 2010.

[5] F. Li, H. Liu, and R. J. Vaccaro, "Performance analysis for DOA estimation algorithms: unification, simplification, and observations," IEEE Transactions on Aerospace and Electronic Systems, vol. 29, no. 4, pp. 1170-1184, 1993.

[6] P. Stoica and A. Nehorai, "MUSIC, maximum likelihood, and Cramer-Rao bound," IEEE Transactions on Acoustics, Speech, and Signal Processing, vol. 37, no. 5, pp. 720-741, 1989.

[7] R. Roy and T. Kailath, "Esprit-estimation of signal parameters via rotational invariance techniques," IEEE Transactions on Acoustics, Speech, and Signal Processing, vol. 37, no. 7, pp. 984-995, 1989.

[8] P. P. Vaidyanathan and P. Pal, "Sparse sensing with co-prime samplers and arrays," IEEE Transactions on Signal Processing, vol. 59, no. 2, pp. 573-586, 2011.

[9] C.-L. Liu and P. P. Vaidyanathan, "Remarks on the spatial smoothing step in coarray music," IEEE Signal Processing Letters, vol. 22, no. 9, pp. 1438-1442, 2015.

[10] W. Zheng, X. Zhang, Y. Wang, M. Zhou, and Q. Wu, "Extended coprime array configuration generating large-scale antenna co-array in massive MIMO system," IEEE Transactions on Vehicular Technology, vol. 68, no. 8, pp. 7841-7853, 2019.

[11] Z. Shi, C. Zhou, Y. Gu, N. A. Goodman, and F. Qu, "Source estimation using coprime array: a sparse reconstruction perspective," IEEE Sensors Journal, vol. 17, no. 3, pp. 755-765, 2017.

[12] J. Shi, G. Hu, X. Zhang, F. Sun, and H. Zhou, "Sparsity-based two-dimensional DOA estimation for coprime array: from sum-difference coarray viewpoint," IEEE Transactions on Signal Processing, vol. 65, no. 21, pp. 5591-5604, 2017.

[13] P. Pal and P. P. Vaidyanathan, "Coprime sampling and the music algorithm," in Proceedings of the 2011 Digital Signal Processing and Signal Processing Education Meeting (DSP/ SPE), Sedona, AZ, USA, January 2011.

[14] C. Zhou, Y. Gu, X. Fan, Z. Shi, G. Mao, and Y. D. Zhang, "Direction-of-arrival estimation for coprime array via virtual array interpolation," IEEE Transactions on Signal Processing, vol. 66, no. 22, pp. 5956-5971, 2018. 
[15] C. Zhou, Y. Gu, Z. Shi, and Y. D. Zhang, "Off-grid directionof-arrival estimation using coprime array interpolation," IEEE Signal Processing Letters, vol. 25, no. 11, pp. 1710-1714, 2018.

[16] C. E. Shannon, "A mathematical theory of communication," Bell System Technical Journal, vol. 27, no. 3, pp. 379-423, 1948.

[17] M. Wax and T. Kailath, "Detection of signals by information theoretic criteria," IEEE Transactions on Acoustics, Speech, and Signal Processing, vol. 33, no. 2, pp. 387-392, 1985.

[18] H. Akaike, "A new look at the statistical model identification," IEEE Transactions on Automatic Control, vol. 19, no. 6, pp. 716-723, 1974.

[19] J. Rissanen, "Modeling by shortest data description," Automatica, vol. 14, no. 5, pp. 465-471, 1978.

[20] L. C. Zhao, P. R. Krishnaiah, and Z. D. Bai, "On detection of the number of signals in presence of white noise," Journal of Multivariate Analysis, vol. 20, no. 1, pp. 1-25, 1986.

[21] L. C. Zhao, P. R. Krishnaiah, and Z. D. Bai, "On detection of the number of signals when the noise covariance matrix is arbitrary," Journal of Multivariate Analysis, vol. 20, no. 1, pp. 26-49, 1986.

[22] L.-C. Zhao, P. Krishnaiah, and Z.-D. Bai, "Remarks on certain criteria for detection of number of signals," IEEE Transactions on Acoustics, Speech, and Signal Processing, vol. 35, no. 2, pp. 129-132, 1987.

[23] D. Xu, X. Yan, S. Xu, H. Luo, J. Liu, and X. Zhang, "Spatial information theory of sensor array and its application in performance evaluation," IET Communications, vol. 13, no. 15, pp. 2304-2312, 2019.

[24] T. Cover and J. Thomas, Elements of Information Theory, John Wiley \& Sons, Hoboken, NJ, USA, 2001.

[25] B. D. Rao and K. V. S. Hari, "Performance analysis of rootmusic," IEEE Transactions on Acoustics, Speech, and Signal Processing, vol. 37, no. 12, pp. 1939-1949, 1989.

[26] C. Liu and P. P. Vaidyanathan, "New Cramér-Rao bound expressions for coprime and other sparse arrays," in Proceedings of the 2016 IEEE Sensor Array and Multichannel Signal Processing Workshop (SAM), Rio de Janeiro, Brazil, July 2016. 